\title{
Utility of Rapid Antigen Detection for Diagnosis of Rota Viral Infection in Children $<2$ Yrs
}

\author{
Shivani Singh*, K. Tukaram Prabhu, Avinash Laghawe, Navinchandra Kaore and Arti Jain \\ People's College of Medical Sciences, Bypass road, Bhanpur, Bhopal 462037, India \\ *Corresponding author
}

\begin{abstract}
A B S T R A C T
Keywords

Rotavirus,

Diarrhoea,

Rapid immuno-

chromatography,

Gastroenteritis,

Infants.

Article Info

Accepted:

12 April 2017

Available Online:

10 May 2017

Rotavirus is a major cause of severe gastroenteritis in young children between 6 months to 2 years of age This study was done with the aim to determine the utility of rapid antigen detection kit for diagnosis of rota viral infection in suspected cases of diarrhoea in children $\leq 2$ yrs. We found that out of the 32 samples, 06 tested postive for the rotaviral antigen by rapid immunochromatography method. $66.7 \%$ of the cases were in 13 - 24 months age group. Detection of Rotavirus infection is necessary in determining the clinical severity as well as finding the prevalence and incidence of this infection in the community. The rapid test kit used is easy to use and inexpensive. This can detect the cases of Rotaviral diarrhoea and be helpful in diagnosis as well as in epidemiological stuides. It can also prevent the unnecessary use of antibiotics. The rapid antigen detection kit for diagnosis of rota viral infection in suspected cases of diarrhoea in children $\leq 2 \mathrm{yrs}$ can be a useful tool.
\end{abstract}

\section{Introduction}

Rota virus is a member of the family Reoviridae, the only RNA virus family that has a double stranded RNA. When observed under electron microscope Rotaviruses have a distinct wheel like appearance. Hence they have been named rota which in Latin means wheel. The virus has a genome of 11 segments of double-stranded RNA, of molecular weight $2 \times 10^{5}$ to $2.2 \times 10^{6}$ Daltons. This RNA is present in the core which is covered with a triple layered capsid (Shobha Broor, 2003). Seven groups (A-G) of Rotaviruses have been described and only groups A, B and C infect humans. Group A has multiple strains and causes most of the childhood diseases.
Worldwide Rotaviruses are a leading cause of acute gastroenteritis in infants and young children worldwide, infecting nearly all children by the age of 5 , often more than once. Each year rotavirus causes approximately 111 million episodes of gastroenteritis, 25 million outpatient visits, and 2 million hospitalizations in children under age 5 worldwide. The incubation period of rotavirus diarrhoea varies from 1-7 days (Parashar et al., 2003; Parashar et al., 2006).

In infants and young children, there is an abrupt onset of severe vomiting and diarrhea with vomiting usually preceding diarrhoea. Stools are usually loose and watery, mucus 
may be present but blood is very rare. Mild to moderate dehydration is seen in 80 per cent of cases and severe loss of fluids and electrolytes may be fatal if untreated. Mild fever is seen in a large majority of cases. The illness usually lasts 3-8 days, but virus shedding continues for about 10 days to 1 month. In immunodeficient children, rotavirus can persist for months. Older children and adults are infected but they generally suffer from subclinical infections and virus is infrequently detected in their stool samples (Steele, 1999).

Rotavirus diarrhoea may show a seasonal variation with a high incidence of the disease in winter months at low relative humidity in north India. Treatment of acute rotavirus infection is nonspecific and involves management of symptoms and, most importantly, maintenance of hydration (Bhautik Modi, 2013).

Transmission of Rotavirus occurs through the feco-oral route (Deepali Masurkar, 2013). Rotavirus is continuously shed in large numbers during the course of disease and stool specimens collected from the first to fourth days of illness are optimal for rotavirus detection (Fischer and Gentsch, 2004). They can be easily identified on electron microscopy of stool samples which is one of the most specific tests for diagnosis. Direct electron microscopy examination of stools for rotavirus has a high sensitivity. However it requires expensive equipment and trained personnel, hence cannot be used in field studies. Other methods like immunoelectro osmophoresis and modified complement fixation test were developed, but they lacked sensitivity.

The recent advent of antigen detection methods based on immunological techniques using monoclonal and polyclonal antibodies has gained attention of researchers. Direct detection of viral antigen by rapid one step immunochromatography technique is an inexpensive, easy to handle sensitive test with no need of invasive procedures and special instrumentation (Sushmita Roy et al., 2008).

It is estimated that 1 in every 250 children born in India dies from rotavirus by the age of 5 yr. India accounts for 17 per cent of the world's estimated rotavirus associated deaths. A number of studies have been conducted on the prevalence of childhood rotavirus diarrhoea in various parts of the country in which rotavirus was detected in $5-71 \%$ of the hospitalized children less than 5 years of age with acute gastroenteritis.

The clinical manifestations of rotavirus diarrhoea alone are not very distinctive to permit exact diagnosis hence testing of samples in the laboratory is the best way to confirm the diagnosis. Most cases of diarrhoea are treated with antibiotics, irrespective of the causative agent.

However, if infection due to rotavirus can be diagnosed early, the misuse and unnecessary usage of antibiotics can be avoided. Etiological diagnosis may not be essential in the treatment of individual patients, but the knowledge of the relative importance and seasonal prevalence of different pathogens in different regions is essential for proper management of outbreaks and for the planning and implementation of control measures.

In this study, stool samples from cases of diarrhoea patients' $\leq 2$ years of age were tested to detect the Rotavirus antigen in the stool specimen by immuno-chromatography test with an aim to know the cause of diarrhoea. If it is of viral origin, unnecessary administration of antibiotics can be prevented and thus help in right and proper management of the patient. 


\section{Materials and Methods}

This cross-sectional prospective study was conducted in a tertiary care hospital of Bhopal and samples were collected from patients attending the pediatric IPD and OPD for a period of Two months - July $15^{\text {th }}$ to September $15^{\text {th }}, 2016$.

Cases were defined as children whose main complaint was acute diarrhea, characterized by occurrence of three or more loose, liquid or watery stools with or without mucous in a 24 hours period. Other symptoms like fever, vomiting etc were also recorded.

SD Bioline kit, Lot no. 14BD0034 Expiry date: 2018/06/29 was used for the test. This kit uses rabbit polyclonal anti-rota virus antibodies which enables identification of Group A Rotavirus antigens.

Stool sample from 32 patients $\leq 2 \mathrm{yrs}$ of age with diarrhoea were collected in a clean wide mouth screw capped bottle. The samples were transported immediately to Microbiology Laboratory.

They were then processed as per instructions of the manufacturer. Briefly, a portion of faeces (about 50g) from a stool sample was taken and the swab provided was placed into the sample collection tube and swirled at least 10 times. The swab was squeezed against the wall of the tube and discarded. The dropping cap was placed on the sample collection tube. 3-4 drops of the prepared sample was dropped into the immuno-chromatography device, the results were read after 10-20 minutes and appearance of test line was taken to be positive.

\section{Results and Discussion}

A total of 32 samples were collected from patients $\leq 2 \mathrm{yrs}$ of age with diarrhea. Out of these 12 patients were from the Out-patient department and 20 were admitted to the Paediatric ward. There were 18 male and 14 female patients with distribution as shown in table 1 .

Out of the 32 samples, $6(18.75 \%)$ were positive for rota viral antigen. Out of the 6 positive cases, $1(16.67 \%)$ was a patient from OPD while $5(83.33 \%)$ were from the ward. Age wise distribution of the cases were as shown in table 2 .

Among the 06 positive cases $03(50 \%)$ were males and $03(50 \%)$ were females and one male among them was an OPD patient.

The average duration of the diarrhoea was 7 days and the average frequency was 6 times a day in our study. Fever was present in all the patients with rota viral diarrhoea with vomitting in $83.3 \%$ (5) of the patients. None of them displayed any signs of dehydration.

Acute gastroenteritis remains a leading cause of post-neonatal under-five mortality in India contributing about $13 \%$ of under-five mortality. Rotavirus is the most important cause for severe gastroenteritis in this age group. Studies in the last decade estimate the annual mortality due to rotavirus in India to be between 90,000 and 153,000 (Jacob John et al., 2014).

Of India's more than 2.3 million annual deaths among children, about 334000 are attributable to diarrhoeal diseases. Rotavirus is the leading cause of severe diarrhoea in children in developed and developing countries. Almost all children have been infected by the time they reach five years of age. In developing countries rotavirus is responsible for approximately half a million deaths per year (Bhautik Modi, 2013). The immune-chromatography test (ICT) used for detection of Rota virus in stool samples give 
rapid results. According to Salwa Badrelsabbha Ibrahim et al., (2015) the ICT is quick, inexpensive, easy to perform and requires very little equipment. Jayoung Kim et al., (2014) have reported that rapid tests show no interference, no cross reactivity, high reproducibility and acceptable agreement rates with other detection technologies like ELISA, ELFA and PCR.

In our study the $18.75 \%$ of the cases tested were positive for rotaviral antigen by rapid immune-chromatography test. This is in concordance with studies by Razaq Hadi Eissa et al., (2014) and Jayoung Kim et al., (2014) However other studies have found higher percentages like Sushmita et al., (2012)- 52.5\%. Hussein (2013) found that distribution of Rotavirus among infants with diarrhea was $50.5 \%$ (52/103). About the distribution of these viruses among age groups, the results show that the most affected age group was 1- 4 months (51.5\%) followed by less than 1 month group which consist $34 \%$.

Table.1 Distribution of the patients - gender wise and OPD/IPD

\begin{tabular}{|l|c|c|}
\hline & Male $(\mathrm{n}=18)$ & Female $(\mathrm{n}=14)$ \\
\hline OPD $(\mathrm{n}=12)$ & 6 & 6 \\
\hline IPD $(\mathrm{n}=20)$ & 12 & 8 \\
\hline Total & 18 & 14 \\
\hline
\end{tabular}

Table.2 Age wise distribution of the cases

\begin{tabular}{|l|l|l|}
\hline Age & Positive $(\mathrm{n}=6)$ & Negative $(\mathrm{n}=26)$ \\
\hline $0-12$ months & $2(33.3 \%)$ & $10(38.5 \%)$ \\
\hline 13-24 months & $4(66.7 \%)$ & $16(61.5 \%)$ \\
\hline
\end{tabular}

Among the 6 patients, 01 patient was from OPD while rests 05 were from paediatric ward, suggesting the seriousness of the disease.

In our study, $4(66.7 \%)$ of the positive cases were in the age group of 13-24 months. Similar result has been reported by Salwa Badrelsabbha Ibrahim et al., (2015) with patients in 6-12 months range having highest rate of rotavirus infection - 54\%. As far as gender distribution was concerned, there was no significant difference in the distribution of patients or in the number of cases, suggesting that the patients of both genders are equally affected by the disease. In contrast to study by Wg Cdr John et al., (2014) whose study showed $33.2 \%$ had fever and $43.6 \%$ had vomiting along with diarrhea and study by
Hussein et al., (2013) had 78.6\% fever and $68 \%$ vomiting symptoms, in our study all the patients presented with fever while 05 patients among the 06 positive cases had vomiting. One patient from OPD did not have vomiting. All the 06 patients were treated symptomatically and all of them recovered without administration of antibiotics.

In a study from Punjab, rotavirus infection has been observed throughout the year with maximum occurrence in November and another peak in the hot and dry months of May (Ram, 1990). The maximum incidence in Pune occurred in winter and the minimum in the rainy season (Kelkar, 1997). This study was conducted during the period of July to September, which can probably explain the low number of positives. Also the number of 
samples tested was 32 and a larger number of samples will have to be tested to make the result statistically significant. This was the limitation of our study.

\section{Acknowledgement}

The authors thank Indian Council of Medical Research (ICMR) for their support for this Short Term Studentship (STS) - 2016 research project.

\section{References}

Bhautik Modi. 2013. Rotavirus diarrhoea current scenario and preventive strategies Guest editorial. Natl. J. Med. Res., 3(2): 104-105.

Deepali, M., Masurkar. 2013. Latex Agglutination Test: A tool for rapid diagnosis of Rotavirus from HIV seropositive and sero-negative patients with diarrhea, Biol. Med., 5: 34-39.

Fischer, T.K. and Gentsch, J.R. 2004. Rotavirus typing methods and algorithms. Rev. Med. Virol., 14: 7182.

Hussein, O.M., Al-Dahmoshi, et al. 2013. Rapid identification of rotavirus, adenovirus and Norovirus using immunochromatography test among Infantile diarrhea, Iraq I.J.S.N., 4(4): 598602.

Jacob John, et al. 2011. Rotavirus gastroenteritis in India, 2011-2013: Revised estimates of disease burden and potential impact of vaccines. Vaccine, 32S: A5-A9.

Jayoung Kim, et al. 2014. Evaluation of an Immunochromatographic Assay for the Rapid and Simultaneous Detection of Rotavirus and Adenovirus in Stool Samples. Ann. Lab. Med., 34: 216-222.
Kelkar, S.D. 1990. Prevalence of human group A rotavirus serotypes in Pune, India (1990-1993). Indian J. Med. Res., 106: 508-12.

Parashar, U.D., Givson, C.J., Bresee, J.S., Glass, R.I. 2006. Rotavirus and severe childhood diarrhea. Emerg. Infect. Dis., 12: 304-306.

Parashar, U.D., Hummelman, E.G., Bresee, J.S., et al. 2003. Global illness and deaths caused by rotavirus disease in children. Emerg. Infect. Dis., 9: 565-572.

Ram, S., Khurana, S., Kusana, S.B., Sharma, S., Vadehra, D.V., Broor, S. 1990. Bioecological factors and rotavirus diarrhoea. Indian J. Med. Res., 91: 16770.

Razaq Hadi Eissa, et al. 2014. Rapid Diagnosis of Rota-Adenoviruses for Acute Gastroenteritis in hospitalized Children under 4 Years Old, Baghdad. Int. J. Curr. Microbiol. App. Sci., 3(1): 453-458.

Salwa Badrelsabbha Ibrahim, et al. 2015. Detection of Rotavirus in children with acute gastroenteritis in Zagazig University Hospitals in Egypt. Electronic Physician, 7: 1227-1233.

Shobha Broor, Dhrubaa Ghosh, Purva Mathur. 2003. Molecular epidemiology of rotaviruses in India. Indian J. Med. Res., 118: 59-67.

Steele, J.C. 1999. Rotavirus. Clin. Lab. Med., 19(3): 691703

Sushmita Roy, et al. 2012. Rapid detection of Rotavirus antigen in stool sample of acute diarrheic children. Bangladesh J. Med. Microbiol., 06(01): 11-13.

Wg Cdr, B.M., John, et al. 2014. Prevalence of rotavirus infection in children below two years presenting with diarrhea. Med. J. Armed Forces India, DOI: http: //dx.doi.org/10.1016/j.mjafi.2014.02.008

\section{How to cite this article:}

Shivani Singh, K. Tukaram Prabhu, Avinash Laghawe, Navinchandra Kaore, Arti Jain. 2017. Utility of Rapid Antigen Detection for Diagnosis of Rota Viral Infection in Children <2 Yrs. Int.J.Curr.Microbiol.App.Sci. 6(5): 1124-1128. doi: https://doi.org/10.20546/ijcmas.2017.605.121 